\title{
Proton transport in polarizable water
}

\author{
S. Walbran and A. A. Kornyshev ${ }^{\text {a) }}$ \\ Institute for Materials and Processes in Energy Systems (IWV-3), Research Center 'Jülich' GmbH, \\ D-52425 Jülich, Germany
}

(Received 12 October 2000; accepted 16 March 2001)

\begin{abstract}
Proton mobility in water determines the conductive properties of water-based proton conductors. We address the problem of proton mobility in pure water using a new, simple, Newtonian molecular dynamics water model which is applicable to proton-rich environments (e.g., polymer electrolyte membranes). This model has degrees of freedom that are "inertial" and "inertialess" relative to the proton. The solvated proton is treated using a local empirical valence bond Hamiltonian, which allows for the efficient simulation of full charge, energy-conserving dynamics in single and multiple-proton systems. The solvated proton displays the Grotthus-type proton transfer mechanism, giving significantly enhanced transport in comparison with the classical diffusion of an $\mathrm{H}_{3} \mathrm{O}^{+}$ion. The model yields an activation energy of $0.11 \mathrm{eV}$, in excellent agreement with experiment. The results are consistent with the observation that nonpolarizable water models, conditioned to reproduce correct values of the static dielectric constant, are predestined to give too large activation energies of proton mobility due to the overweighted spectrum of the slower nuclear modes.

(C) 2001 American Institute of Physics. [DOI: 10.1063/1.1370393]
\end{abstract}

\section{INTRODUCTION}

Water is a proton conductor. However, the intrinsic conductivity of distilled water is miserable due to a negligible density of free, mobile protons of dissociation. The mobility of protons in water is nonetheless very high. Thus, whenever an excess proton is donated to water, it runs fast.

This feature is fundamental to reactions in acids, enzymatic catalysis, operation of proton pumps in living cells, and for proton transport through polymer electrolyte membranes. The latter is crucial in the realm of technical applications, primarily for low temperature fuel cells. ${ }^{1}$ In such a system, the proton conducting polymer electrolyte membrane material, being also a kind of (polymeric) acid, absorbs water with consequent dissociation of acidic groups of side chains. This generates a high concentration of "free" protons in water-filled channels, resulting in a high conductivity of the membrane at a sufficient water content.

The magnificent feature of water as a medium for proton transfer is its democratic attitude to an excess proton, which after "birth" becomes exchangeable with each of the remaining protons in water. It is not the same excess proton that hops from one water molecule to another; instead, a much more efficient, relay, "Grotthus" mechanism of proton transport takes place. ${ }^{2}$ That the Grotthus transport prevails over the classical $\mathrm{H}_{3} \mathrm{O}^{+}$ion diffusion has become commonplace, but how this actually proceeds remained to be a subject of big debates. ${ }^{3}$ Only recently, after a series of papers, ${ }^{4-7}$ has a more or less coherent view on the qualitative features of the proton transport in water emerged, as discussed in the next section.

While the methods used in these works are scientifically excellent, they are for a number of reasons, described in

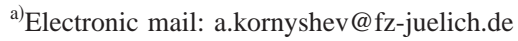

detail below, inappropriate or prohibitively expensive to apply in more general systems of interest. In addition, the fundamental "purity" of the ab initio methods makes it difficult to extract information about the broader physical principles governing the microscopic behavior: Why is the proton transport that fast? What forms the Franck-Condon barrier for the elementary act of proton transfer? Is the observed, relatively moderate, activation free energy (near $0.1 \mathrm{eV}$ ) compatible with the mechanism of the elementary act?

An attempt to answer these questions, as well as a thirst for a molecular dynamics (MD) proton transport simulation program applicable to a cell with a large number of molecules and simultaneous transport of several protons, has motivated our study. Such model water could later be poured into any relevant environment (biological, technical) in order to follow the effect of the medium on proton conductance, including in particular an environment which itself may donate protons upon water uptake and hydration.

The objective of the MD model of proton transport in pure water is to reproduce, as well as possible, equilibrium water structure, self-diffusion of water molecules, preexponential and activation factors of charge mobility, infrared spectra, etc. A reasonable reproduction of these properties will help ensure that, using standard atom-atom potentials for the interaction of water with the environment, we will be able to predict, at least qualitatively, the environmental effect on the proton mobility. A success along these lines will open a door for tracing changes in proton conductivity upon systematic chemical and structural modifications of the environment.

After a brief review of the current picture of proton transport in water and the reported achievements in MD simulations, we formulate our model and simulation scheme. We then present the results for structural characteristics of our model, and for the Grotthus proton dynamics in compari- 
son with the dynamics of a model $\mathrm{H}_{3} \mathrm{O}^{+}$ion and with experimental data. We find that this model of water, involving "inertialess" electronic polarizability reducing FranckCondon barriers, yields an activation barrier compatible with the observed activation free energy of proton mobility.

The model we present here is a new model of water with high frequency polarizability, in which a solvated proton can move via structural diffusion. The elementary act is based on the hopping of a proton from a hydronium ion to a neighboring water molecule. The model has, yet, a number of shortcomings: protons still do not move fast enough, while water behavior is too lively. These and other deficiencies are discussed in the end of the paper along with ways of possibly improving the model.

\section{CURRENT VIEW ON PROTON TRANSPORT IN WATER}

\section{A. Qualitative picture}

The solvated proton has been envisioned in a number of ways. First, the excess proton could be part of an $\mathrm{H}_{3} \mathrm{O}^{+}$ion, in which all three protons are equivalent. Second, it could reside in a Zundel ${ }^{8} \mathrm{H}_{5} \mathrm{O}_{2}^{+}$complex with a proton between two water molecules, binding them in a cluster. Third, it could be pictured as an Eigen ${ }^{9} \mathrm{H}_{9} \mathrm{O}_{4}^{+}$cluster, which consists of an $\mathrm{H}_{3} \mathrm{O}^{+}$ion and three strongly bound $\mathrm{H}_{2} \mathrm{O}$ molecules, each attached to one of the protons in $\mathrm{H}_{3} \mathrm{O}^{+}$. One can, of course, imagine other intermediate structural arrangements; in any case, all of the clusters are apparently short lived in water, and their life and disappearance are crucial parts of the proton transport dynamics. The generic structural diffusion pathways of the three species are pictured as follows.

In the most simplistic view, the excess proton hops from a $\mathrm{H}_{3} \mathrm{O}^{+}$donor site to any neighboring water acceptor molecule, leaving a neutral water molecule behind. The proton hop is preceded by suitable configurational and polarization fluctuations in water; such fluctuations determine the transition state. In order for the proton transfer to occur, the electronic overlap between the donor and acceptor which triggers the transfer must, moreover, be strong enough; this is only possible when the donor and acceptor acquire the proper coordination, resembling an $\mathrm{H}_{5} \mathrm{O}_{2}^{+}$cluster. At the distance between the two oxygen atoms close to those of the equilibrium ground state of the $\mathrm{H}_{5} \mathrm{O}_{2}^{+}$cluster in the gas phase (2.4 $\AA$ ), the interaction between the $\mathrm{H}_{3} \mathrm{O}^{+}$and $\mathrm{H}_{2} \mathrm{O}$ moieties is so strong that there is no barrier for the proton motion along the $\mathrm{O}-\mathrm{H}-\mathrm{O}$ coordinate, and the proton is delocalized between the two water molecules. ${ }^{10,4}$ The equilibrium $\mathrm{O}-\mathrm{O}$ distance may be slightly larger in the condensed phase, leaving a small barrier, and a shallow double well may emerge.

A second view, based on infrared spectral data, is one in which the $\mathrm{H}_{3} \mathrm{O}^{+}$ion is a less stable molecular entity than the $\mathrm{H}_{5} \mathrm{O}_{2}^{+}$complex. ${ }^{8} \mathrm{H}_{5} \mathrm{O}_{2}^{+}$would then be the basic state of the proton and the $\mathrm{H}_{5} \mathrm{O}_{2}^{+}$structural defect would move together with its charge in water. Again, this does not involve classical defect diffusion as a whole, but structural diffusion based proton transfer dominates and gives the high proton transport mobility. A water molecule, next to such a cluster, fluctuates into a configuration where it forms a new $\mathrm{H}_{5} \mathrm{O}_{2}^{+}$together with the nearest molecule of the original $\mathrm{H}_{5} \mathrm{O}_{2}^{+}$. This also requires orientational adjustment and deformation of both adjacent and remote water molecules in the original $\mathrm{H}_{5} \mathrm{O}_{2}^{+}$ cluster. Subsequent fluctuations move remote water molecules out of the initial $\mathrm{H}_{5} \mathrm{O}_{2}^{+}$configuration, shifting the charge by one water molecule. This motion may proceed along any of the four external protons of the $\mathrm{H}_{5} \mathrm{O}_{2}^{+}$cluster.

Fluctuational preorganization followed by virtually barrierless proton transfer carries over to mechanisms based on the $\mathrm{H}_{9} \mathrm{O}_{4}^{+}$cluster. This is followed by decoupling of one of the peripheral molecules in the cluster, shifting the center of the cluster in the direction of the incoming acceptor water molecule. The process runs along one of the six peripheral protons.

Common to these three simplistic views is that $\mathrm{H}^{+}-n \mathrm{H}_{2} \mathrm{O}$ clusters self-assemble spontaneously up to a certain size. If a cluster were disrupted by the configurational fluctuations of the same molecule whose fluctuational motion created the cluster, there would be no charge transfer. If, however, the disruption were to come from one of those molecules which was not part of the initial cluster, the charge would be shifted. In the class of models discussed above, the activation free energy of cluster motion is of the same order as for breaking a hydrogen bond.

Recent Kohn-Sham density functional simulations for molecular wires ${ }^{11}$ have shown that $\mathrm{H}_{3} \mathrm{O}^{+}$and $\mathrm{H}_{5} \mathrm{O}_{2}^{+}$clusters are the dominant defects in water. Ab initio calculations in multimolecular water systems $\mathrm{s}^{4}$ demonstrated the presence of these and larger clusters in more or less equal amounts. The particular cluster size may be important for proton conductivity of water in a confined geometry: squeezing water inside a membrane pore or embedding charged polymer side chains may also change the distribution balance in the contribution of clusters of different size, since their condensed state energies are similar. In other words, the spatial constraints on the pathways via clusters of different size may affect the proton mobility.

\section{B. Proton transfer in molecular dynamics simulations}

From a simulation standpoint, one of the greatest challenges to describing the transfer of a proton in a Newtonian simulation is the conservation of the full charge of the ion, due to the near-universal use of partial charges in molecular dynamics models.

One approach to describing the solvated proton is to treat all protons equivalently, resulting in a fully dissociable water model. In order to conserve charge, however, each particle must carry its full formal charge, ${ }^{12,13}$ the partial charges must vary in some way with coordination, ${ }^{14}$ or one must perform some sort of electronic structure calculation during the dynamics. ${ }^{4}$ While a fully dissociable model is a very desirable goal, those which are available in the literature exhibit a number of undesirable features, such as poor agreement with experimental transport properties, ${ }^{13}$ or extreme computational complexity. ${ }^{4}$

An alternative line of attack is to denote a subset of the protons in the sample as "special," embedded in a bath of more simple model water molecules. In such a case, the 
charge conservation laws can be applied in a localized region around a special proton, reducing the complexity of the model significantly. If no opportunity is provided to exchange a special proton with background water protons, ${ }^{15,16}$ the transport is limited to the non-Grotthus diffusion of a unique entity, resembling that of a light $\mathrm{Li}^{+}$. However, if the identity of the special proton can be dynamically switched, the interesting features of the Grotthus mechanism can be included in the simulation (with the exception of only solitonlike multiproton transfers, which are not expected to play a significant role in the disordered liquid water environment ${ }^{17}$ ).

Recently, empirical valence bond (EVB) methods have been used to achieve proton exchange in such a manner. ${ }^{5-7}$ In these studies, the partial charges and interaction potentials of the solvated cluster are obtained from the lowest energy state of a Hamiltonian matrix consisting of standard interaction potentials and empirical off-diagonal elements. The model we present here is similar in many respects, but differs in key ways, allowing the efficient, full-charge, energyconserving simulation of multiple-proton systems, including solvent polarization effects.

In Ref. 5, diffusion of the proton was not allowed to occur, since the proton is permanently bound between two special host water molecules. Thus, the model is not applicable for the measurement of long-time transport properties.

In Ref. 6, energy conservation was not obtained. In addition, proton diffusion was measured using a coordinate denoted the "charge barycenter," which, being in fact the polarization, can exhibit large fluctuations in "position" due simply to reorientation of charged species, and hence could give rise to exaggerated apparent diffusion coefficients. Finally, the requirement of 15-20 EVB states per proton prohibits the model's use in multiproton systems, as detailed below.

In Ref. 7, the authors carry out an extensive study with an elaborate EVB model within a nonpolarizable water model. They note that nonpolarizable water models, whose partial charges are chosen to reproduce the enhanced condensed phase dipole moment, overestimate the polarization response to a solvated ion, and compensate by applying an ad hoc correction factor of $c=0.76$ to the Coulomb interactions of the solvated proton with the water environment; in effect, their solvated proton does not carry the full formal charge of $+1 e$. Their exquisite, but expensive, path-integral treatment of the quantum nature of the nuclear modes yields very deep insight and extremely valuable results, but, in combination with the requirement of 8-10 EVB states per proton, prohibits the efficient application of this model in multiproton environments.

\section{POLARIZABLE WATER}

We introduce a flexible model for water based on the nonpolarizable Toukan-Rahman model, ${ }^{18}$ with an implementation of high-frequency dielectric response which mimics electronic polarizability within a classical simulation. ${ }^{19}$ Our approach avoids the complications of point dipoles or variable charges which have been used in other polarizable water models. ${ }^{20,12,13}$ Our model requires no self-consistency

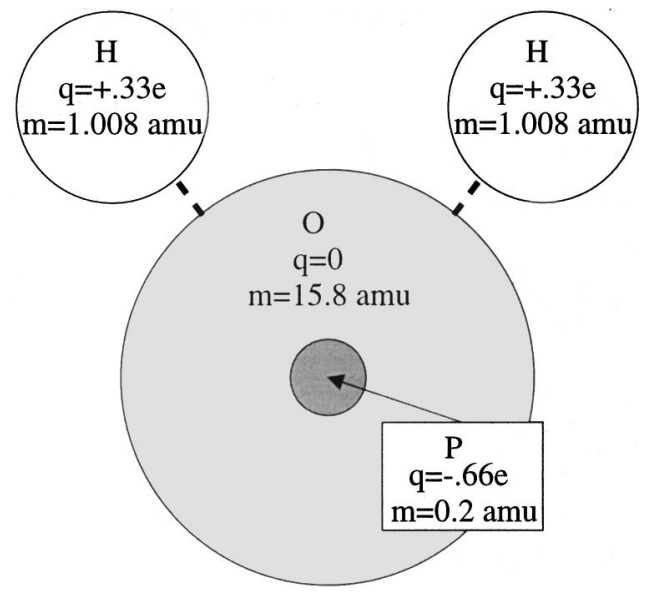

FIG. 1. The four sites of the water molecule are shown schematically, along with their respective charges and masses.

loops on polarization variables, therefore allowing efficient computation. In addition, by implementing the polarizability with point charges (instead of point dipoles), we are also able to use standard Ewald methods for long-range electrostatics.

The water molecule in our model has four interaction sites, diagrammed in Fig. 1, each of which undergoes Newtonian dynamics in the MD simulation. Two of these sites correspond to the protons in the water molecule, each having charge $q_{\mathrm{H}}=+0.33 e$ and mass $m_{\mathrm{H}}=1.008 \mathrm{amu}$. These are bound to each other and to a chargeless "oxygen" site with mass $m_{\mathrm{O}}=16.00 \mathrm{amu}-m_{P}$ via the intramolecular interactions of the Toukan-Rahman water model, which effectively reproduce the vibrational spectrum of water. Explicitly, this interaction potential is given by

$$
\begin{aligned}
V_{\text {mol }}= & \frac{1}{2} a\left[\left(\Delta r_{1}\right)^{2}+\left(\Delta r_{2}\right)^{2}\right]+\frac{1}{2} b\left(\Delta r_{3}\right)^{2} \\
& +c\left(\Delta r_{1}+\Delta r_{2}\right) \Delta r_{3}+d\left(\Delta r_{1} \Delta r_{2}\right),
\end{aligned}
$$

where $\Delta r_{1}$ and $\Delta r_{2}$ are the stretches of $\mathrm{O}-\mathrm{H}$ bond lengths $\left(r_{\mathrm{OH}}-r_{\mathrm{OH}}^{0}\right)$, and $\Delta r_{3}$ is the stretch of the $\mathrm{H}-\mathrm{H}$ bond $\left(r_{\mathrm{HH}}\right.$ $\left.-r_{\mathrm{HH}}^{0}\right)$. The chargeless oxygen site is bound to a fourth site, with mass $m_{\mathrm{P}}=0.20 \mathrm{amu}$ and charge $q=-2 q_{\mathrm{H}}$, by an interaction of the form

$$
V\left(r_{\mathrm{OP}}\right)=\frac{1}{2} k_{2} r_{\mathrm{OP}}^{2}+\frac{1}{4} k_{4} r_{\mathrm{OP}}^{4} .
$$

The dynamics of this low-mass charged site provides an "inertialess" polarizability, intended to mimic the electronic polarizability of the frequency domain higher than the $\mathrm{O}-\mathrm{H}$ stretch frequency, in a manner very similar to that of the Car-Parinello methods ${ }^{21}$ most often used in first-principles dynamic simulations. The charge values were chosen to give the correct gas phase dipole moment of the water molecule, with the polarization force constants and mass chosen to give good results for the structure of condensed phase water and to set the polarization response frequency region; no attempt to reproduce the complex shape of the spectrum in this essentially quantum domain was made.

Intermolecular interactions consist solely of the Coulomb interactions between each pair of charged particles, and the Lennard-Jones 12-6 potential between oxygens

$$
V_{\mathrm{LJ}}\left(r_{\mathrm{OO}}\right)=A_{\mathrm{LJ}} / r_{\mathrm{OO}}^{12}-B_{\mathrm{LJ}} / r_{\mathrm{OO}}^{6}
$$


TABLE I. Parameters of the water model.

\begin{tabular}{ll}
\hline \hline$q_{\mathrm{H}}$ & $+0.33 e$ \\
$q_{\mathrm{O}}$ & 0 \\
$q_{\mathrm{P}}$ & $-0.66 e$ \\
$k_{2}$ & $110.08 \mathrm{kcal} \mathrm{mole}^{-1} \AA^{-2}$ \\
$k_{4}$ & $2444.26 \mathrm{kcal} \mathrm{mole}^{-1} \AA^{-4}$ \\
$a$ & $9.331 \mathrm{mdyn}^{-1} \mathrm{a}$ \\
$b$ & $2.283 \mathrm{mdyn}^{-1 \mathrm{a}}$ \\
$c$ & $-1.469 \mathrm{mdyn}^{-1 \mathrm{a}}$ \\
$d$ & $0.776 \mathrm{mdyn}^{-1} \mathrm{a}$ \\
$r_{\mathrm{OH}}^{0}$ & $1.000 \AA^{\mathrm{a}}$ \\
$r_{\mathrm{HH}}^{0}$ & $1.633 \AA^{\mathrm{a}}$ \\
$A_{\mathrm{LJ}}$ & $1895.38\left(e^{2} / \AA^{12} \AA^{12 \mathrm{a}}\right.$ \\
$B_{\mathrm{LJ}}$ & $1.884\left(e^{2} / \AA\right) \AA^{6} \mathrm{a}$ \\
\hline
\end{tabular}

${ }^{a}$ Reference 18.

from the Toukan-Rahman model. Parameters for the water model potentials are given in Table I.

\section{THE HYDRATED PROTON}

We consider a simplified model in which the solvated proton consists of a hydronium $\left(\mathrm{H}_{3} \mathrm{O}^{+}\right)$entity which may exchange a proton with a neighboring $\mathrm{H}_{2} \mathrm{O}$. In this model, the hydronium interacts with other molecules (water or other hydronium) via Coulomb interactions and an oxygenoxygen Lennard-Jones potential; the latter is taken to have the same parameters as for the water alone. However, proton exchange proceeds by "mixing the identities" within the $\mathrm{H}_{3} \mathrm{O}^{+}-\mathrm{H}_{2} \mathrm{O}$ transferring cluster, as described in detail below. The model thus rests on the picture of an isolated hydronium ion and a dynamic hydronium-water interaction.

\section{A. The isolated hydronium}

The isolated $\mathrm{H}_{3} \mathrm{O}^{+}$model consists of five interaction sites. Three of these sites correspond to the protons in the hydronium, each having charge $q_{\mathrm{H}}=+0.33 e$ and mass $m_{\mathrm{H}}$ $=1.008 \mathrm{amu}$, as in the water molecule. These three sites are bound to each other and to an "oxygen" site, with mass $m_{\mathrm{O}}=16.00 \mathrm{amu}-m_{\mathrm{P}}$, via the intramolecular potentials from the EVB model of Ref. 7. These are a Morse $\mathrm{O}-\mathrm{H}$ potential

$$
V_{\mathrm{OH}}\left(R_{\mathrm{OH}}\right)=C_{\mathrm{OH}}\left(1-e^{-a_{\mathrm{OH}}\left(R_{\mathrm{OH}}-R_{\mathrm{OH}}^{0}\right)}\right)^{2}
$$

and a harmonic $\mathrm{H}-\mathrm{O}-\mathrm{H}$ angular potential

$$
V_{\mathrm{HOH}}(\alpha)=\frac{1}{2} k_{\alpha}\left(\alpha-\alpha_{0}\right)^{2} \text {. }
$$

The oxygen site is bound to a "polarization" site exactly as in the water model described above, i.e., via Eq. (1). The oxygen site and polarization site carry the same masses as in the water molecule. The polarization site carries the same charge as in water; thus, in order to maintain the net $+1 e$ charge of the hydronium, the hydronium oxygen site carries a nonzero charge $q_{\mathrm{O}}$, in contrast with the chargeless water oxygen site. (See Fig. 2.)

The choice of charge on the proton sites, in combination with the parameters of the polarization degrees of freedom, has a profound influence on hydrogen bonding. The choice to have equal charges on the proton sites in both the isolated water and hydronium molecules was made for a number of reasons. Not least is the desire to treat the solvated proton on

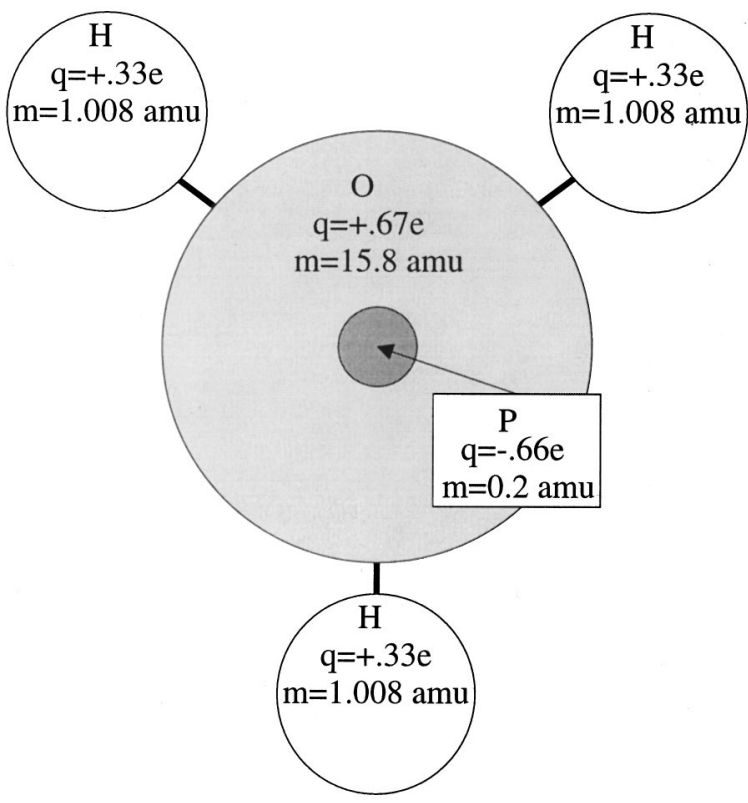

FIG. 2. The five sites of the hydronium model are shown schematically, along with their respective charges and masses.

as equal footing as possible as the background protons. Practically, this choice also simplifies the charge switching functions, as described in the next section. Primarily, however, the choice was determined by the fact that we found we could achieve good small cluster and bulk structural and energetic results with this choice.

\section{B. The mixing of charges: switching function}

At any given time in the simulation, the solvated hydronium is partnered, in our model, with a neighboring water molecule to form a cluster in which proton transfer may take place, denoted hereafter the "transferring cluster.' Although defined stoichiometrically as $\mathrm{H}_{5} \mathrm{O}_{2}^{+}$, this cluster is not limited to the Zundel cation structure, as described below.

Within a single transferring cluster, we take the partial charges on the hydronium and partner water molecule to be a function of the proton transfer coordinate

$$
\mathcal{Q} \equiv\left|R_{\mathrm{O} * \mathrm{H}}\right|-\left|R_{\mathrm{OH}}\right|,
$$

where $\mathrm{O}^{*}$ and $\mathrm{O}$ are the oxygen sites of the hydronium and water molecule, respectively (similar functions have been used in Refs. 5, 41 and elsewhere). Because we have chosen the hydrogen and polarization site charge to be the same in both the water and hydronium molecules, the charge switching takes place only on the oxygen sites. These dynamic charges are given in terms of the isolated water and hydronium model charges via

$$
\begin{aligned}
& \tilde{q}_{\mathrm{O} *}=[1-f(\mathcal{Q})] q_{\mathrm{O} *}+f(\mathcal{Q}) q_{\mathrm{O}}, \\
& \widetilde{q}_{\mathrm{O}}=[1-f(\mathcal{Q})] q_{\mathrm{O}}+f(\mathcal{Q}) q_{\mathrm{O}} * .
\end{aligned}
$$

The function $f(\mathcal{Q})$ is taken to be equal to 0 for $\mathcal{Q}<-\mathcal{Q}_{0}$, and equal to 1 for $\mathcal{Q}>+\mathcal{Q}_{0}$. For $\mathcal{Q}$ in the range $-\mathcal{Q}_{0}<\mathcal{Q}$ $<\mathcal{Q}_{0}$, the function $f(\mathcal{Q})$ is taken to be a polynomial which smoothly varies between value 0 at $\mathcal{Q}=-\mathcal{Q}_{0}$ and value 1 for $\mathcal{Q}=+\mathcal{Q}_{0}$; explicitly, 


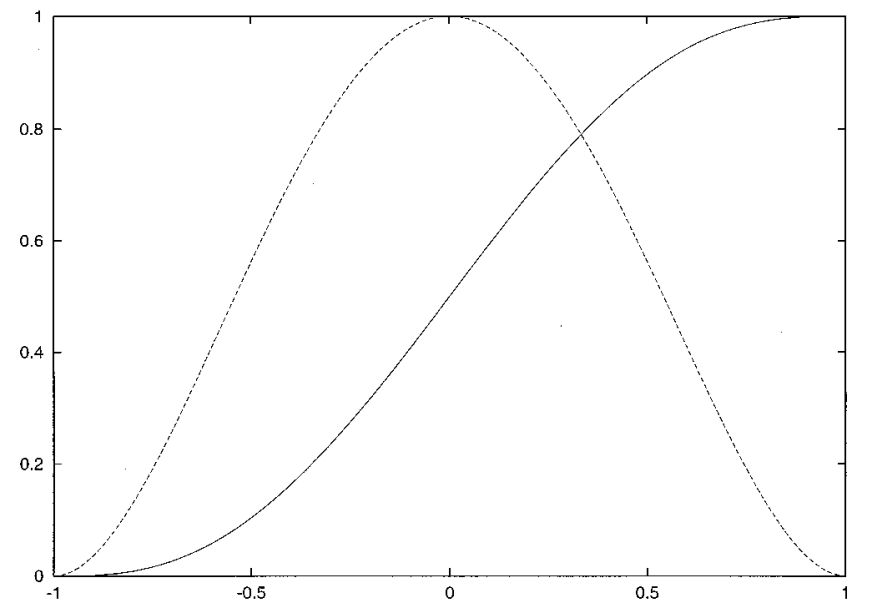

FIG. 3. The form of the charge switching polynomial $f(\mathcal{Q})$ (solid) and the matrix element polynomial $\Lambda(\mathcal{Q})$ (dashed) are shown, with parameters $\Lambda_{0}$, $\mathcal{Q}_{0}$, and $\mathcal{Q}_{1}$ set to 1 for display purposes.

$$
f(\mathcal{Q})=\frac{1}{2}+\frac{\alpha}{5}\left(\frac{\mathcal{Q}}{\mathcal{Q}_{0}}\right)^{5}-\frac{2 \alpha}{3}\left(\frac{\mathcal{Q}}{\mathcal{Q}_{0}}\right)^{3}+\alpha\left(\frac{\mathcal{Q}}{\mathcal{Q}_{0}}\right)
$$

with $\alpha=15 / 16$ required to obtain the \pm 1 limits, and $\mathcal{Q}_{0}$ a parameter which determines the length scale of the switching function. A plot of $f(\mathcal{Q})$ is shown Fig. 3.

The use of a charge switching function requires, for the conservation of energy, the calculation of forces on particles due to these extra degrees of freedom. That is, the force on particle $j$ becomes

$$
F_{j}=-\frac{\partial V}{\partial x_{j}}-\sum_{k}\left(\frac{\partial V}{\partial q_{k}}\right)\left(\frac{d q_{k}}{d f}\right)\left(\frac{d f}{d \mathcal{Q}}\right)\left(\frac{d \mathcal{Q}}{d x_{j}}\right)
$$

with the summation technically over all other sites $k$; however, since, by the rules of the game, $d q_{k} / d f$ is zero for all particles outside the transferring cluster, this calculation is in fact not particularly expensive.

\section{The mixing of potentials: matrix elements and diagonalization}

Within the cluster, the interaction potentials are mixed according to a diagonalization procedure very similar to that used in a global EVB model. We take the interaction energy to be the lowest surface of the matrix

$$
\left[\begin{array}{cc}
V_{i}(\{R\}) & \Lambda(\mathcal{Q}) \\
\Lambda(\mathcal{Q}) & V_{f}(\{R\})
\end{array}\right]
$$

Here $i$ and $f$ stand for the "initial" and "final" states at a fixed set of positions $\{R\}$ : In the initial state, $V_{i}$ is the energy of the cluster calculated under the assumption that the sites in the molecule which is "water" interact as in the water model, while the "hydronium", sites interact as in the hydronium model; in the final state, the molecule which is "water' is combined with the transferring proton and undergoes hydronium-model interactions, whereas the molecule labeled "hydronium" is separated from its proton and undergoes water-model interactions. These situations are outlined in Fig. 4.
"Initial" state
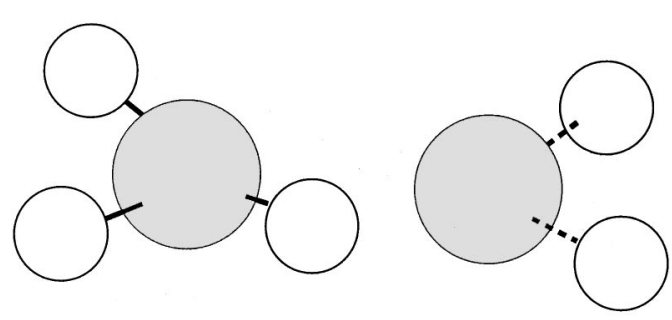

"Final" state

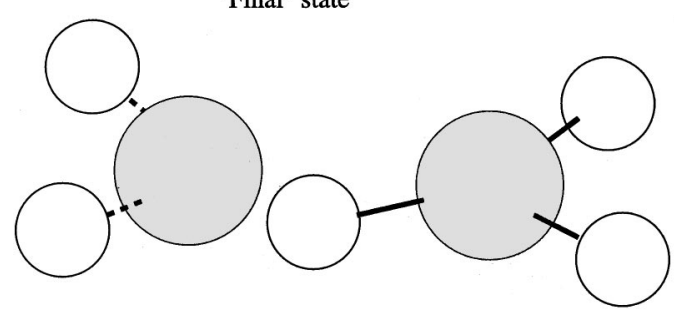

FIG. 4. The "initial" and "final" states of the transferring cluster Hamiltonian are shown schematically. Interactions of the hydronium model are schematically indicated using solid lines, with those of the water by dashed lines.

$\Lambda(\mathcal{Q})$ is an empirical matrix element, which we take to be a function of the proton transfer variable $\mathcal{Q}$ defined in Eq. (2). The function $\Lambda(\mathcal{Q})$ is taken to be equal to 0 for $\mathcal{Q}<$ $-\mathcal{Q}_{1}$ and $\mathcal{Q}>+\mathcal{Q}_{1}$. For $\mathcal{Q}$ in the range $-\mathcal{Q}_{1}<\mathcal{Q}<\mathcal{Q}_{1}$, the function $\Lambda(\mathcal{Q})$ is taken to be a polynomial which smoothly goes to value 0 at $\mathcal{Q}= \pm \mathcal{Q}_{1}$; explicitly,

$$
\Lambda(\mathcal{Q})=\Lambda_{0}\left[\left(\frac{\mathcal{Q}}{\mathcal{Q}_{1}}\right)^{4}-2\left(\frac{\mathcal{Q}}{\mathcal{Q}_{1}}\right)^{2}+1\right]
$$

with $\Lambda_{0}$ and $\mathcal{Q}_{1}$ parameters which set the strength and width of the matrix element. A plot of $\Lambda(\mathcal{Q}) / \Lambda_{0}$ is shown Fig. 3 .

We wish to emphasize that the interactions of the charged sites within the transferring cluster with the sites of molecules external to the cluster are fully determined by the procedure described in the preceding section, and that the matrix diagonalization described here involves only local bonding interactions. It is this "locality" of the charge switching function which allows our model to be efficiently used in multiproton environments. In contrast, in a full, "global" EVB model, charges on the sites in a transferring cluster are determined by a matrix diagonalization procedure in which the matrix elements depend on the charge state of other transferring clusters in the system. This is naturally a more precise description, but in a multiproton system the use of the global EVB framework requires an expensive selfconsistency loop at each time step. Avoiding it means an approximation (of uncontrolled accuracy) similar to the quasichemical (Bethe) approximation in statistical physics.

\section{The partnering process}

The solvation shell of the hydronium ion consists normally of three water molecules, as shown in Fig. 5. In the model, each of these waters is a potential receptor of a proton from a hydronium, and, once a proton has been transfered, the waters neighboring the newly formed hydronium become potential receptors, thus allowing full, democratic diffusion 


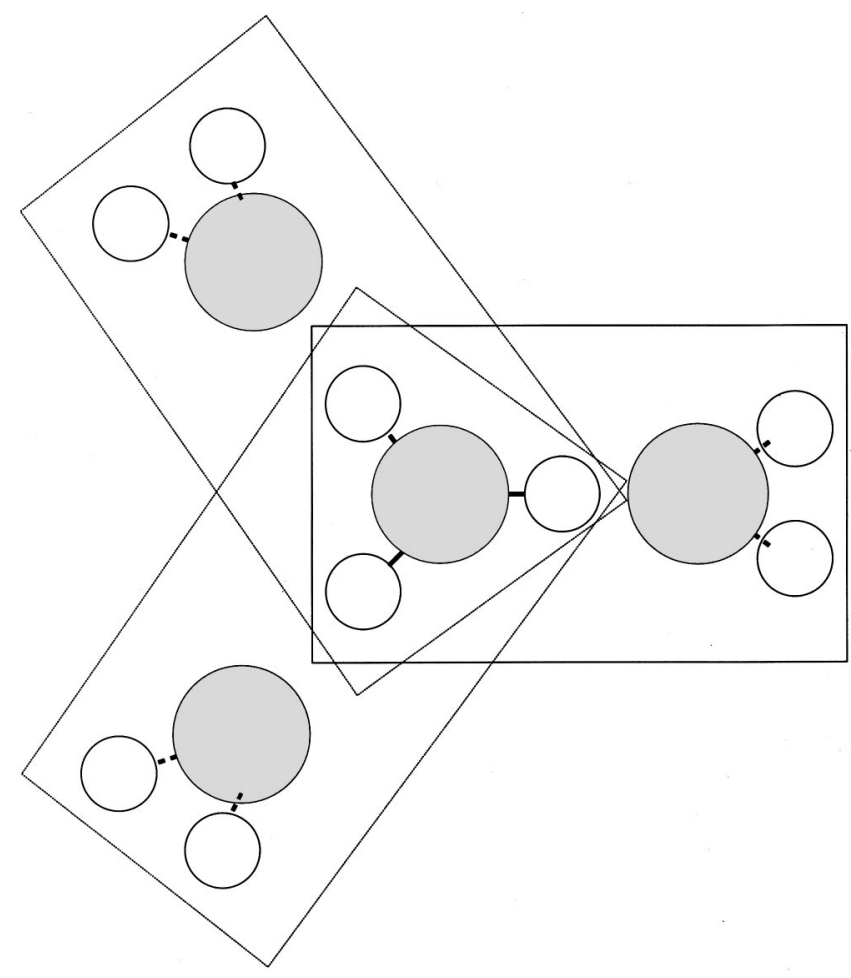

FIG. 5. A hydronium ion (center) is shown solvated by three water molecules. The solid rectangle indicates the hydronium-water pair forming a "transferring cluster." The dotted rectangles indicate the alternative clusters which would form during the simulation if their partnering criteria were to become more favorable, as discussed in the text.

of the solvated proton. However, only one proton in a given proton transfer cluster may be in the transferring process at a given time: we have no means of accounting for virtual $\mathrm{OH}^{-}$ states. In general, we assume that the water molecule closest to the hydronium, i.e., with the shortest oxygen-oxygen distance, is the most likely candidate where the proton can be transferred to, and this molecule is assigned as a partner. When the dynamically changing values of $\Lambda$ and $f$ as a function of proton transfer coordinate $\mathcal{Q}$ are nonzero, however, switching the partner would lead to a discontinuous interaction potential, and hence a loss of energy conservation. Therefore, we restrict partner switching to the times when these values reside at zero. This is equivalent to saying that partner switching can only take place in a transferring cluster configuration corresponding to a "pure" hydronium ion and water molecule.

In practice, the partnering proceeds as follows: A solvated proton is introduced into a bath of water molecules in the form of an $\mathrm{H}_{3} \mathrm{O}^{+}$ion. The three nearest-neighbor waters are identified, and the closest water is taken as the partner to form the transferring cluster. The simulation proceeds under the dynamics of the interaction potentials described above. If the hydronium proton facing the partner water is successfully transferred, that partner water molecule becomes a hydronium entity in the simulation, and its neighboring waters (including the former hydronium) are now identified as potential partners. If the transferring cluster fluctuates instead to a configuration where $f(\mathcal{Q})$ and $\Lambda(\mathcal{Q})$ are zero, i.e., the transferring proton returns to its host hydronium, the three
TABLE II. Parameters of the proton model.

\begin{tabular}{ll}
\hline \hline$q_{\mathrm{H}}$ & $+0.33 e$ \\
$q_{\mathrm{O} *}$ & $+0.67 e$ \\
$q_{\mathrm{P}}$ & $-0.66 e$ \\
$C_{\mathrm{OH}}$ & $266.3 \mathrm{kcal} \mathrm{mol}^{-1 \mathrm{a}}$ \\
$a_{\mathrm{OH}}$ & $1.285 \AA^{-1 \mathrm{a}}$ \\
$R_{\mathrm{OH}}^{0}$ & $0.98 \AA^{\mathrm{a}}$ \\
$k_{\alpha}$ & $73.27 \mathrm{kcal} \mathrm{mol}^{-1} \mathrm{rad}^{-2 \mathrm{a}}$ \\
$\alpha^{0}$ & $116.0 \mathrm{deg}^{\mathrm{a}}$ \\
$\Lambda_{0}$ & $3.10 \mathrm{eV}$ \\
$\mathcal{Q}_{0}$ & $0.50 \AA$ \\
$\mathcal{Q}_{1}$ & $0.55 \AA$ \\
\hline
\end{tabular}

${ }^{\mathrm{a}}$ Reference 7.

possible partners are re-evaluated, and the nearest water is chosen as the next partner.

This process gives a well-defined procedure through which the solvated proton may be exchanged with background protons and undergo energy-conserving Grotthus transport. However, these simplifying assumptions in which only one proton undergoes potential transfer at any given time not only might reduce the observed transport properties, but also introduce more fundamental physical issues of asymmetry in the treatment of the solvated and background protons and hysteresis of the potential energy surface. The model is correct only to the extent that the effects of these assumptions are small.

\section{E. Parameter choices}

Generally, the length scales $\mathcal{Q}_{0}$ and $\mathcal{Q}_{1}$ for the switching function and matrix element, respectively, need to be sufficiently large to give a smooth transition in the interactions during proton transfer. However, the requirement that $f(\mathcal{Q})$ and $\Lambda(\mathcal{Q})$ be zero when switching partners constrains these parameters: the length scales must be short enough for such configurations to not be so rare that they limit transport. The size of $\mathcal{Q}_{0}$ relative to $\mathcal{Q}_{1}$ has been adjusted to obtain overall smoothness in interactions during a transfer. The final free parameter, the matrix element magnitude $\Lambda_{0}$, is the determining factor in the energy of the $\mathrm{H}_{5} \mathrm{O}_{2}^{+}$small cluster but has negligible effect on that of the $\mathrm{H}_{9} \mathrm{O}_{4}^{+}$cluster, in which no proton is transferring. $\Lambda_{0}$ was adjusted to give reasonable structure and formation energies for small $\left(\mathrm{H}^{+}\right)\left(\mathrm{H}_{2} \mathrm{O}\right)_{n}$ clusters, and to maximize the bulk proton transfer rate at $300 \mathrm{~K}$ given the other constraints.

\section{SIMULATION DETAILS}

Simulations are performed in an NVE ensemble using the Verlet algorithm, with equilibration to the desired temperature carried out by occasional velocity rescaling during preliminary molecular dynamics runs. We present results for systems containing 100 water molecules ("pure water"), as well as for systems containing 99 water molecules and one hydronium molecule. In all cases, the cell is a cube with side length $14.41 \AA$, long-range electrostatics are calculated using standard Ewald methods, ${ }^{22}$ and the simulation time step is 0.25 femtosecond. For the "hydrated proton" system, parameters for the proton transfer are given in Table II, allow- 
ing the proton to be exchanged with solvating "partner" molecules as described above. For "classical $\mathrm{H}_{3} \mathrm{O}^{+}$-ion", systems, the transferring cluster mixing parameters $\Lambda_{0}, Q_{0}$, and $Q_{1}$ are set to zero to prohibit proton transfer, while retaining the pure hydronium dynamics. Statistical averaging was carried out by performing eight parallel simulation runs starting from independent initial conditions for runs of 50150 picoseconds each, giving a total averaging time of $0.4-$ 1.2 nanosecond per system. Calculations were carried out on a Cray T3E of the von Neumann Institute of Applied Mathematics at the Research Center "Juelich."

\section{RESULTS AND DISCUSSION}

\section{A. Structure}

Figure 6 shows the radial distribution functions calculated in a simulation cell containing only water, at temperature $300 \mathrm{~K}$. It can be seen that the structure of water in our model is very well maintained with respect to its unpolarizable parent, the Toukan-Rahman model. ${ }^{18}$ This is demonstrated, for example, in the first oxygen-oxygen peak remaining at $2.8 \AA$ and the first intermolecular oxygenhydrogen peak being obtained at $1.75 \AA$; also, key coordination numbers are obtained at the appropriate distances. A difference worth remarking upon, however, is that the oxygen-oxygen peak appears somewhat broadened in the current work, which is a sign of enhanced water mobility, as detailed below.

In Table III we report on the structure and formation energies of the prototypical small protonated clusters $\mathrm{H}_{5} \mathrm{O}_{2}^{+}$ and the threefold-symmetric $\mathrm{H}_{9} \mathrm{O}_{4}^{+}$. The results obtained are in quite reasonable agreement with ab initio and other calculations considering the simplicity of the model. ${ }^{19,7}$ In Fig. 7, we show the radial distribution function for water solvating the hydronium molecule at $300 \mathrm{~K}$, with proton hopping disabled as described above. (Such a plot is difficult to make with hopping enabled, due to the exchange of species' identities inherent in the Grotthus mechanism.) The hydronium is correctly solvated by three water molecules, with the oxygen-oxygen peak near $2.55 \AA$.

\section{B. Kinetic properties}

We calculate the self-diffusion coefficient for water molecules, for the classical $\mathrm{H}_{3} \mathrm{O}^{+}$ion, and the solvated proton. For computational convenience (due to the relatively large number of water molecules in the simulation), the diffusion coefficient of water was calculated using the oxygen velocity autocorrelation function as

$$
D=\frac{1}{3} \int_{0}^{\infty}\langle\vec{v}(0) \vec{v}(t)\rangle d t,
$$

where $\langle\cdots\rangle$ is an ensemble average. The diffusion coefficient of the classical $\mathrm{H}_{3} \mathrm{O}^{+}$and proton were more conveniently measured using the positions via

$$
D=\lim _{t \rightarrow \infty} \frac{\left\langle\Delta \vec{x}^{2}\right\rangle}{6 t} .
$$
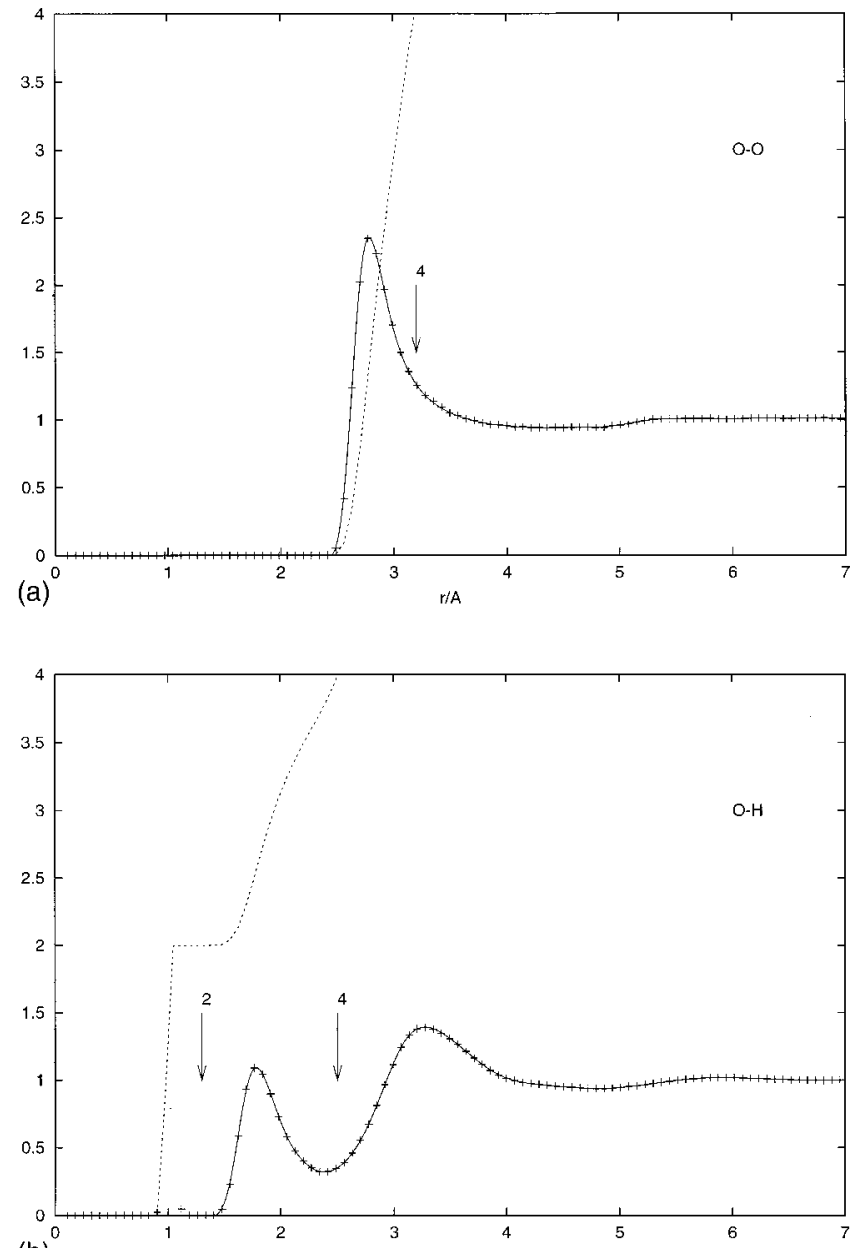

(b)

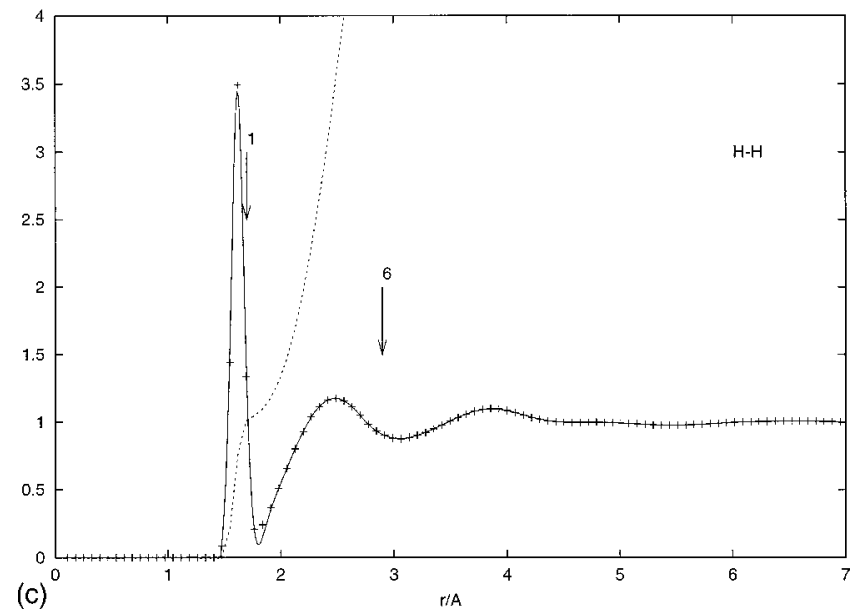

FIG. 6. The radial distribution functions (a) $g_{\mathrm{OO}}(r)$ for oxygen-oxygen, (b) $g_{\mathrm{OH}}(r)$ oxygen-hydrogen, and (c) $g_{\mathrm{HH}}(r)$ hydrogen-hydrogen are shown for the model water at $300 \mathrm{~K}$. The strong intramolecular $\mathrm{O}-\mathrm{H}$ peak in (b) has been removed for display purposes. Solid lines show smooth curves for the simulation data (points), while dotted lines indicate the running integration number. Arrows indicate the positions at which key coordination numbers are obtained. (Compare with Fig. 1 in Ref. 18.)

While the coordinate of the classical $\mathrm{H}_{3} \mathrm{O}^{+}$needs no explanation, it must be defined for the solvated proton. Here we use the coordinate

$$
\vec{x}=[1-f(Q)] \vec{x}_{\mathrm{O}^{*}}+f(Q) \vec{x}_{O}
$$


TABLE III. Formation energies and distances obtained for small clusters using the model.

\begin{tabular}{cccc}
\hline \hline & $E\left(\mathrm{kcal} \mathrm{mole}^{-1}\right)$ & $R_{\mathrm{OO}}(\AA)$ & $R_{\mathrm{OH}}(\AA)$ \\
\hline $\mathrm{H}_{5} \mathrm{O}_{2}^{+}$ & 30.6 & 2.41 & 1.205 \\
$\mathrm{H}_{9} \mathrm{O}_{4}^{+}$ & 68.3 & 2.55 & 1.008 \\
\hline \hline
\end{tabular}

to describe the position, where $f(Q)$ is the charge switching function defined in Eq. (3) This definition corresponds to the position of the host oxygen in the case of a hydroniumlike configuration, and gives a smooth interpolation towards the receptor oxygen for intermediate values of $f$. (In contrast with the "charge barycenter" polarization coordinate of Ref. 6 , our coordinate choice, always lying between the two oxygens, is not subject to exaggerated apparent motion due merely to polarization rearrangement, and hence gives a more realistic picture of the position of the transferring proton.) The diffusion coefficient was found to converge well (i.e., $\left\langle\Delta \vec{x}^{2}\right\rangle / 6 t$ becoming constant) within a 25 picosecond averaging window.

In Fig. 8, we show the simulation results for the diffusion coefficient for each species as a function of absolute temperature $T$, along with fits to the Arrhenius form

$$
D=D_{0} e^{-E / k_{B} T} .
$$

The activation energies $E$ obtained from these fits are given in Table IV. It can be seen that, due to the Grotthus hopping, the transport of the solvated proton is significantly faster than that of the classical diffusion of the $\mathrm{H}_{3} \mathrm{O}^{+}$ion. The activation energy for the proton, at $0.11 \mathrm{eV}$, is in excellent agreement with the experimental result of $0.107 \mathrm{eV}$ for this temperature range. ${ }^{23}$ However, we note that the absolute value of the proton diffusion coefficient is approximately half of that which is obtained experimentally, while the diffusion of water itself is significantly higher than that of real water; the activation energy for water, at $0.07 \mathrm{eV}$, is also lower than the

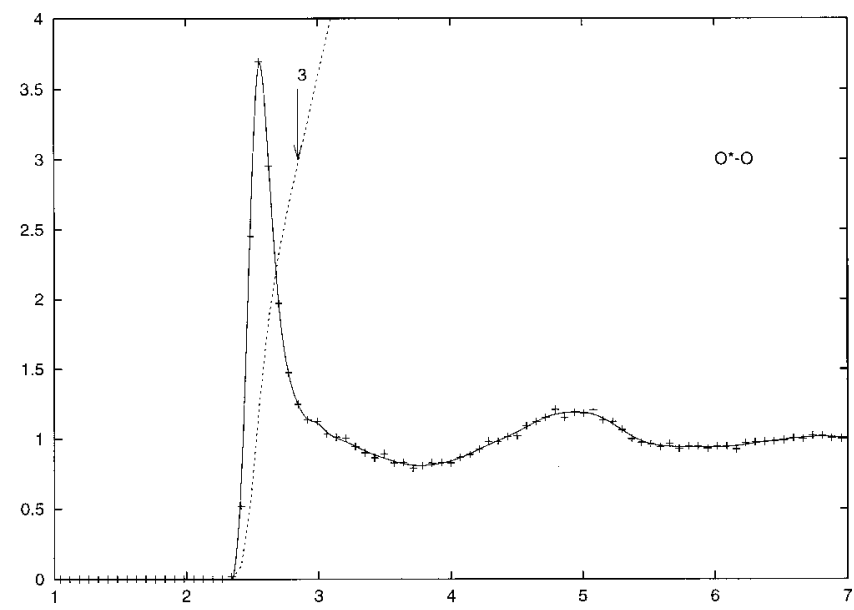

FIG. 7. The hydronium-water oxygen-oxygen radial distribution function $g_{\mathrm{O} * \mathrm{O}}(r)$ and the corresponding integrated coordination number is shown for the hydronium ion at $300 \mathrm{~K}$. The arrow indicates the position $\left(R_{\mathrm{O} * \mathrm{O}}\right.$ $=2.85 \AA$ ) at which the coordination number 3 is obtained.
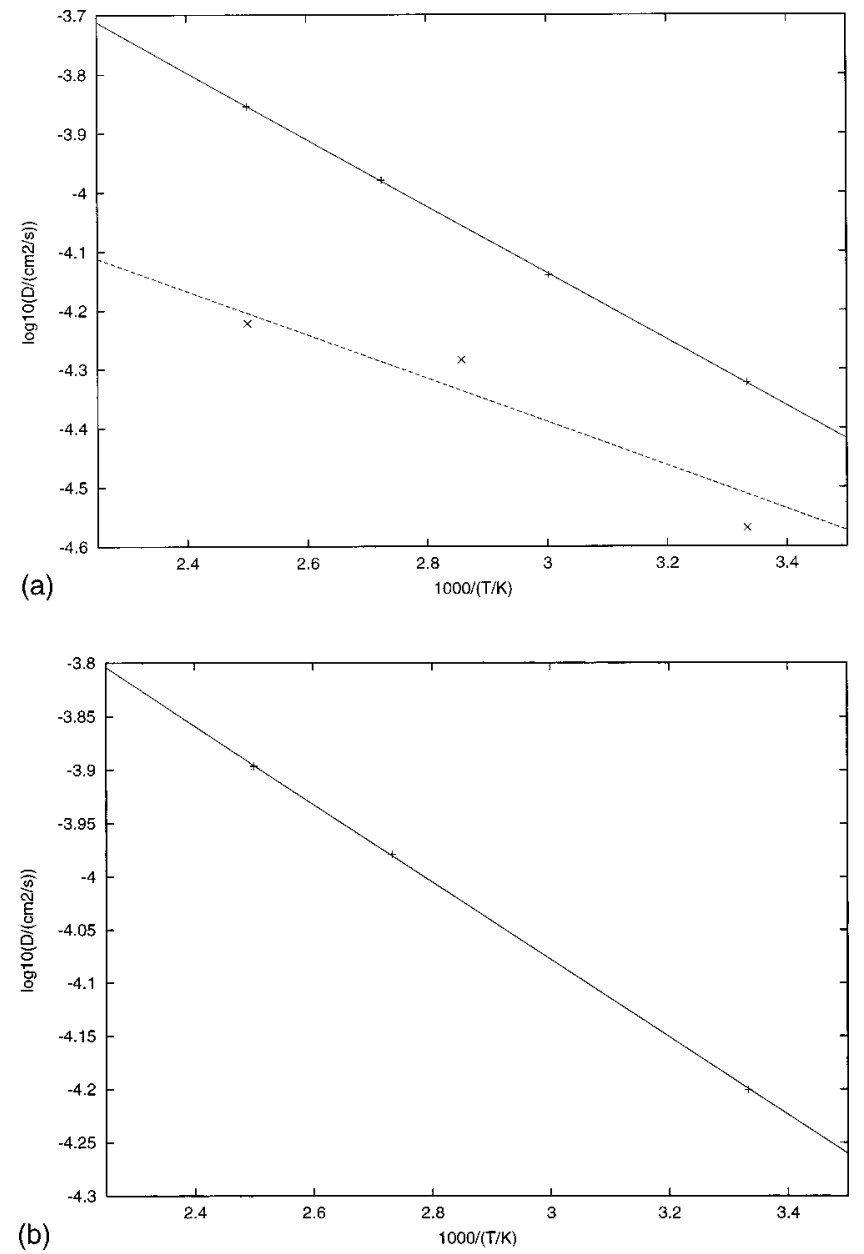

FIG. 8. (a) An Arrhenius plot of the self-diffusion coefficients $D$ of the solvated proton (crosses, data; fit, solid line) and the classical $\mathrm{H}_{3} \mathrm{O}^{+}(\times$, data; fit, dashed line) as measured in simulation. Proton hopping gives a clear enhancement to the motion of the solvated charge. The activation energy for the solvated proton agrees with the experimental value, but the absolute value is lower than expected (Ref. 23). (b) The same plot as in (a), but for the water molecule. The diffusion coefficient for water is larger, and activation energy lower, than that obtained in experiment (Ref. 23). The corresponding activation energies for all species are given in Table IV.

experimental value of $0.170 \mathrm{eV} .{ }^{23}$ We discuss these discrepancies and potential solutions for them in more detail in the conclusion section.

We calculate infrared spectra for the system using the current-current autocorrelation function, where the current is defined as

$$
\vec{J}=\sum_{i}\left(q_{i} \frac{d \vec{x}_{i}}{d t}+\frac{d q_{i}}{d t} \vec{x}_{i}\right),
$$

the second term being nonzero with the varying charges inside a proton transfer cluster. In Fig. 9, we plot the Fourier

TABLE IV. Diffusion activation energies for $300 \mathrm{~K}<T<400 \mathrm{~K}$.

\begin{tabular}{lcc}
\hline \hline & Simulation & Experiment $^{\mathrm{a}}$ \\
\hline Proton & $0.11 \mathrm{eV}$ & $0.107 \mathrm{eV}$ \\
Hydronium & $0.07 \mathrm{eV}$ & \\
Water & $0.07 \mathrm{eV}$ & $0.170 \mathrm{eV}$ \\
\hline \hline
\end{tabular}

${ }^{\mathrm{a}}$ Reference 23 . 


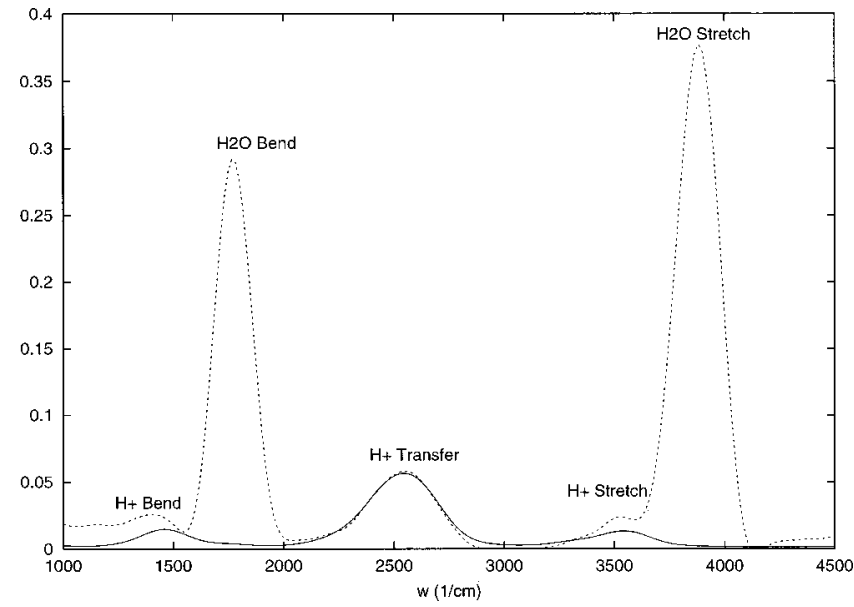

FIG. 9. We plot the infrared spectra $\langle J(0) J(t)\rangle_{\omega} / \omega$ for the solvated proton system using the total current $J$ (dashed line) and the current $J_{\mathrm{H}}$ from the transferring cluster alone (solid line), as discussed in the text. Peaks labeled " $\mathrm{H} 2 \mathrm{O}$ ', correspond to water frequencies, while peaks labeled " $\mathrm{H}+$ ", correspond to those of species in the transferring cluster.

transform of the autocorrelation function $\langle\vec{J}(0) \vec{J}(t)\rangle_{\omega} / \omega$ with $\vec{J}$ being the total current in the sample. For comparison, we also plot the data using only the current $\vec{J}_{\mathrm{H}}$, in which we count only the current due to the motion of the molecules within the transferring cluster, in order to highlight the spectral structure due to the solvated proton. It can be seen that the vibrational spectrum for water maintains the good agreement with the parent Toukan-Rahman model. The solvated charge gives, in addition to the expected hydronium peaks near 1500 and $3500 \mathrm{~cm}^{-1}$, a broad peak between 2000 and $3000 \mathrm{~cm}^{-1}$ due to the transferring proton. We note that frequency-dependent conductivity of the sample may be obtained from such data via the Kubo formula, ${ }^{24}$ but also that in this high-frequency region, quantum corrections must be made to symmetrize the response. ${ }^{25-27}$ Given the simplified, classical nature of the model, we have not carried out such a detailed analysis here.

\section{CONCLUSION}

We have presented a model for the solvated proton which is simple and efficient, yet able to reproduce the features which make aqueous proton transport differ from classical ionic diffusion. Water molecules are flexible and polarizable, without sacrificing the simplicity of point charges for long-range electrostatic calculations, nor introducing expensive self-consistency loops, nor requiring non-Newtonian dynamics. The solvated proton is implemented in a manner which allows the transport of the full formal ionic charge, while conserving charge and energy. The "local" nature of the algorithm allows for the efficient simulation of multipleproton systems. The proton displays a significantly enhanced diffusion over the classical, vehicle-assisted diffusion of hydronium, which can only be attributed to hopping, Grotthusmechanism transport. The activation energy of proton diffusion was able to be measured by direct simulations at various temperatures, and is found to be in excellent agreement with experiment.
There are, however, some caveats with the model. In particular, the high diffusion constant of the water indicates that there is room for improvement in our parametrization. It is very likely that, in combination with the (unchanged) intermolecular Lennard-Jones parameters from the ToukanRahman model, the shift of weight of the electrostatic response from low to high frequency region due to the polarization has reduced the activation energy for water transport. We expect that the polarization and intermolecular interaction parameters can be modified to improve the behavior of the water model without destroying the successes of the solvated proton model, and look forward to doing so.

The proton transport, while clearly enhanced by proton hopping, remains in absolute value somewhat lower than that obtained experimentally. To some extent this may be due to the simplicity of the proton hopping model, in particular the requirement that changing partner water molecules only occurs when the charge switching function $f(Q)$ and matrix element $\Lambda(Q)$ are zero. Extending the model to allow simultaneous fractional charge and potential mixing with more than one neighboring water, without requiring the explicit modeling of the $\mathrm{OH}^{-}$anion, is possible, though perhaps cumbersome; this is, however, the natural path via which charge distribution over larger regions (i.e., $\mathrm{H}_{9} \mathrm{O}_{4}^{+}$) can be incorporated. The latter will offer a larger number of entrance and exit terminals in the proton transferring cluster, which could increase the pre-exponential factor in the proton mobility, while retaining the same, correct value of the activation energy. In addition, quantum tunneling effects and corrections due to zero point vibration energy levels for the protons in local wells which are expected to increase the proton hopping rate, ${ }^{7,28-33}$ have not been explicitly considered here.

Our results are consistent with the contemporary theory of proton transfer in condensed media, ${ }^{34-43}$ in which the Franck-Condon factor determines the activation energy of proton mobility. Namely, if the contribution from the slow nuclear modes of water is misleadingly large in an MD model, the model will not be able to give a correct value of the activation energy. Separation between the fast and slow degrees of freedom with respect to the moving proton helps to understand which modes do form the Franck-Condon barrier. MD models of water which do not have fast, "inertialess" polarizability typically overweigh the spectrum of the slow modes, being intended, e.g., to reproduce the correct value of dielectric constant, with no explicit account of electronic degrees of freedom involved. Such models are predestined to give an incorrectly large contribution to the activation energy.

In future work, we will further tune and extend the model to better reproduce the water dynamics and the preexponential factor of proton diffusion, and look forward to applying it to the proton-rich environment of proton exchange membrane systems for which the model was designed.

\section{ACKNOWLEDGMENTS}

We are thankful to M. Eikerling, J. W. Halley, A. M. Kuznetzov, D. Stöver, D. Stolten, and J. Ulstrup for many 
useful discussions. One of us (S.W.) is thankful to the Alexander von Humbolt Foundation for the fellowship which supported this research. This work was carried out on the T3E and RS60000 computers at the ZAM Institute of the Research Center "Juelich."

${ }^{1}$ Proton Conducting Membrane Fuel Cells, edited by S. Gottesfeld, T. F. Fuller, Proceedings Vol. 98-27 (The Electrochemical Society, Inc., Pennington, 1999).

${ }^{2}$ C. J. D. von Grotthus, Ann. Chim. (Paris) LVIII, 54 (1806)

${ }^{3}$ N. Agmon, J. Phys. Chem. 100, 1072 (1996).

${ }^{4}$ D. Marx, M. E. Tuckerman, J. Hutter, and M. Parinello, Nature (London) 397, 601 (1999).

${ }^{5}$ D. E. Sagnella and M. E. Tuckermann, J. Chem. Phys. 108, 2073 (1998).

${ }^{6}$ R. Vuilleumier and D. Borgis, J. Chem. Phys. 111, 4251 (1999).

${ }^{7}$ U. W. Schmidt and G. A. Voth, J. Chem. Phys. 111, 9361 (1999).

${ }^{8}$ G. Zundel and H. Metzger, Z. Phys. Chem. (Munich) 58, 225 (1968).

${ }^{9}$ E. Wicke, M. Eigen, and Th. Ackermann, Z. Phys. Chem. (Munich) 1, 340 (1954).

${ }^{10}$ K. D. Kreuer, Chem. Mater. 8, 610 (1996).

${ }^{11}$ R. R. Sadeghi and Hai-Ping Cheng, J. Phys. Chem. 111, 2086 (1999).

${ }^{12}$ F. H. Stillinger and C. W. David, J. Chem. Phys. 69, 1473 (1978).

${ }^{13}$ J. W. Halley, J. R. Rustad, and A. Rahman, J. Chem. Phys. 98, 4110 (1993).

${ }^{14}$ C. W. David, J. Chem. Phys. 104, 7255 (1996).

${ }^{15}$ X.-D. Din and E. M. Michaelides, AIChE J. 44, 35 (1998).

${ }^{16}$ J. Ennari, M. Elomaa, and F. Sundholm, Polymer 40, 5035 (1999)

${ }^{17}$ Proton Transfer in Hydrogen Bonded Systems, edited by T. Bountis, NATO ASI Series, Vol. 291 (Plenum, New York, 1992).

${ }^{18}$ K. Toukan and A. Rahman, Phys. Rev. B 31, 2643 (1985).

${ }^{19}$ For example, D. Wei and D. R. Salahub, J. Chem. Phys. 106, 6086 (1997); L. Ojamäe, I. Shavitt, and S. J. Singer, ibid. 109, 5547 (1998).

${ }^{20}$ For example, P. Alhström, A. Wallqvist, S. Engström, and B. Jönsson, Mol. Phys. 14, 513 (1989); M. Sprik and M. L. Klein, J. Chem. Phys. 89,
7556 (1988); S.-B. Zhu, S. Singh, and G. W. Robinson, ibid. 95, 2791 (1991); G. Corongiu, Int. J. Quantum Chem. 42, 1209 (1992).

${ }^{21}$ R. Car and M. Parinello, Phys. Rev. Lett. 55, 2471 (1985).

${ }^{22}$ S. W. de Leeuw, J. W. Perram, and E. R. Smith, Proc. R. Soc. London, Ser. A 373, 27 (1980).

${ }^{23}$ K. D. Kreuer, in Proton Conductors: Solids, Membranes, and Gels: Materials and Devices, edited by P. Colomban (1992), p. 474.

${ }^{24}$ R. Kubo, J. Phys. Soc. Jpn. 12, 570 (1957).

${ }^{25}$ P. Schofield, Phys. Rev. Lett. 4, 239 (1960).

${ }^{26}$ P. A. Egelstaff, Adv. Phys. 11, 203 (1962).

${ }^{27} \mathrm{Ph}$. A. Bopp, A. A. Kornyshev, and G. Sutmann, J. Chem. Phys. 109, 1939 (1998)

${ }^{28}$ R. P. Bell, The Tunnel Effect in Chemistry (Chapman and Hall, Oxford, 1980).

${ }^{29}$ Proton Transfer Reactions, edited by E. F. Caldin and V. Gold (Chapman and Hall, London, 1975).

${ }^{30}$ A. G. Kresge, Acc. Chem. Res. 23, 43 (1990).

${ }^{31}$ For a modern review, see in A. M. Kuznetsov and J. Ulstrup, Can. J. Chem. 77, 1085 (1999).

${ }^{32}$ A. M. Kuznetsov, Charge Transfer in Physics, Chemistry and Biology (Gordon and Breach, Amsterdam, 1995), Chap. 6.

${ }^{33}$ A. M. Kuznetsov and J. Ulstrup, Electron Transfer in Chemistry and Biology (Wiley, Chichester, 1999), Chap. 10.

${ }^{34}$ R. R. Dogonadze, A. M. Kuznetsov, and V. G. Levich, Electrochim. Acta 13, 1025 (1968)

${ }^{35}$ R. A. Marcus, J. Phys. Chem. 72, 891 (1968); 72, 4249 (1968).

${ }^{36}$ Yu. I. Kharkats, J. Res. Inst. Catal., Hokkaido Univ. 26, 15 (1978).

${ }^{37}$ A. M. Kuznetsov, J. Electroanal. Chem. 180, 121 (1984).

${ }^{38}$ H. Sumi and R. A. Marcus, J. Chem. Phys. 84, 4894 (1986).

${ }^{39}$ H. Sumi and J. Ulstrup, Biochim. Biophys. Acta 955, 26 (1988).

${ }^{40}$ D. Borgis and J. T. Hynes, J. Chem. Phys. 94, 3619 (1991); Chem. Phys. 170, 315 (1993); J. Phys. Chem. 100, 1118 (1996).

${ }^{41}$ D. Borgis, G. Tarjus, and H. Azzouz, J. Chem. Phys. 97, 1390 (1992).

${ }^{42}$ R. J. Cukier, J. Phys. Chem. 98, 2377 (1994).

${ }^{43}$ J.-Y. Fang and S. Hammes-Schiffer, J. Chem. Phys. 106, 8442 (1997). 